\title{
Influencia de los procesos sensoriomotores y ambientales sobre el comportamiento adaptativo y la atención
}

Resumen

Martha Mafía Tobón

Terapeuta Ocupacional Universidad Nacional de Colombia Especialista en Docencia Universitaria.

Colegio Mayor del Rosario

Email: awadtobon $@$ hotmail.com

Por un mañana.

Transversal $62 \mathrm{~N}^{\circ} 174-51$

San José de Bavaria.

Bogotá, D. C., Colombia
Este artículo identifica y describe la influencia de los procesos sensoriomotores y ambientales sobre el comportamiento adaptativo y la capacidad para atender, requisitos indispensables para un desempeño ocupacional exitoso. Muestra la influencia del desarrollo en estos procesos. Identifica la relación entre estos, el ambiente y las habilidades para atender y responder adecuadamente al entorno.

Explica que cuando el proceso de Integración Sensorial no es eficiente se pueden presentar problemas de Atención. Muchos autores como Mulligan en 1998,Cermak en 1988, Holborow y Oetter en 1986, han estudiado la relación entre los problemas de integración sensorial y los de Atención. Estos planteamientos también fueron comprobados por Fisher y Murray quienes proponen que los problemas de atención son el resultado de un deficiente procesamiento de la información sensorial: Táctil, Vestibular, Propioceptiva, Visual y Auditiva.

Esta propuesta se apoya especialmente en las teorías sobre desarrollo sensoriomotor, integración sensorial y aprendizaje de autoras como Ayres, Fisher, Bundy, Mulligan, y Kranowitz.

Este artículo también propone la evaluación de los prerrequisitos sensoriomotores y del ambiente. Esta se basa en la experiencia de la autora y del grupo de trabajo del Jardín Infantil Por un Mañana, ubicado en Bogotá, Colombia. 
La propuesta de evaluación consiste en:

Una lista de chequeo cuyo objetivo es identificar los comportamientos que caracterizan el desempeño sensoriomotor y ejecutivo del niño.

Lla cbservación sistemática de los ambientes con la elaboración de cuadros que permitan describir lo que se ve cuando se observa al niño durante la ejecución de sus actividades en los contextos en que se desempeña.

Un cuestionario ambiental que permite conocer las características y posibilidades del ambiente físico y de personas de la casa con el fin de establecer la necesidad de hacer cambios y las opciones con que se cuenta.

\section{Palabras claves}

Desarrollo y problemas de Atención. Integración Sensorial y Atención. Comportamiento Adaptativo y atención. Desarrollo sensoriomotor y ejecutivo. Evaluación del desempeño sensoriomotor y la atención.

Nicolás está en clase de arte. La profesora indica a los niños que deben pintar líneas horizontales llenando la hoja. El no la está escuchando pues se está tratando de limpiar la mano que está untada de pintura ino le gusta como se siente! Además está preocupado porque el asiento de arte es muy alto y no se siente seguro, mejor se coge con una mano. ¿Se caerá y todo el curso se echará a reír como en la clase pasada? Sus compañeros empiezan a trabajar, así que él intenta empezar. ¿Qué tal unas flores? Cuando la profesora ve su trabajo le llama la atención ya que eso no es lo que hay que hacer. Nicolás la mira asombrado, ¿cuando dijo eso de las líneas? Pero no puede reiniciar su trabajo y por eso tira el frasco de tempera sobre el trabajo. Su profesora lo reprende por el "descuido" y lo saca del salón. Nicolás prefiere el castigo. Por fin esta libre de la tortura de untarse y sostenerse en esa silla tan alta.

Nicolás puede ser el típico niño con problemas para responder adecuadamente a las demandas ambientales. Tiene problemas de integración sensorial que afectan su capacidad para atender y plantea pobres respuestas para adaptarse a la situación.

El objetivo de este artículo es identificar y describir la influencia de los procesos sensoriomotores y de ejecución, sobre el Comportamiento Adaptativo y la ca- 
pacidad para atender y proponer estrategias de evaluación de estos procesos.

Primero, se mostrará la influencia del desarrollo de los procesos de Integración Sensorial sobre el comportamiento adaptativo y la capacidad para atender. Luego se describirá la relación entre el ambiente, los procesos sensoriomotores y de ejecución y las habilidades para atender y responder adecuadamente al entorno. Después se describirán algunas evaluaciones del desempeño del niño y del entorno que se utilizan en terapia ocupacional. Finalmente se mostrará la propuesta para realizar la evaluación de los procesos sensoriomotores y de ejecución que influyen sobre el comportamiento adaptativo y la atención.

Esta propuesta se apoya especialmente en las teorías sobre desarrollo sensoriomotor, Integración Sensorial y aprendizaje de autoras como Ayres, Fisher, Bundy, Mulligan, y Kranowitz.

La evaluación se basa en la experiencia de la autora y del grupo de trabajo del Jardín Infantil Por un Mañana, ubicado en Bogotá, Colombia. Propone emplear estrategias para identificar el perfil sensoriomotor y de ejecución del niño y conocer el ambiente en que este se desempeña. Así se posibilitará un abordaje que contemple todas las áreas que necesitan ser atendidas.

\section{Influencia de los procesos de Integración Sensorial sobre el Comportamiento Adaptativo y los periodos de atención}

Muchos autores como Jean Ayres, Anne Fisher y Anita Bundy, Winnie Dunn, Carol Stock Kramowitz, entre otros, han definido el Comportamiento Adaptativo (CA). Por ejemplo Fisher y Bundy (6) plantean que el hombre es capaz de responder en forma adecuada a las demandas ambientales gracias a la habilidad que tiene de reconocer, relacionar, e interpretar la información proveniente de su entorno.

Esta información se recibe a través de los sentidos y es procesada en el Sistema Nervioso Central por los mecanismos de Integración Sensorial (IS) y Representación Cortical. A partir de esos procesos cerebrales se produce una respuesta, generalmente de tipo motor, adecuada a cada situación.

EI CA permite al individuo una armónica interacción con el ambiente para desempeñar en forma eficiente habilidades motoras, comunicativas, cognitivas y ejecutivas. Asi este puede lograr un desempeño ocupacional exitoso para aprender, jugar, trabajar o descansar (Kielhofner, 1994).

Las personas reciben información sensorial todo el tiempo. Esta información táctil, vestibular, propioceptiva, visual $y$ auditiva es relacionada e interpreta- 
da en su cerebro produciendo una respuesta adaptativa acorde con la situación (Ayres, 1979. Fisher, 1991El proceso para producir el CA se puede explicar con el ejemplo de un niño tomando un dictado, que se encuentra a continuación:

- Escoge atender únicamente al profesor y a su trabajo a pesar de otros estímulos ambientales (Orientación - atención).

- Mira la hoja (información visual).

- Toca el papel con el borde de su mano y siente la forma del lápiz (información táctii).

- Mantiene la posición sentado y el adecuado agarre del lápiz (información vestibular y propioceptiva).

- Escucha al profesor que está dictando las palabras (información auditiva).

- Reúne la información sensorial proveniente del aula de clase y de su cuerpo y la organiza para ser interpretada (IS).

- Relaciona lo que escucha con el conocimiento que tiene de la palabra, su significado y forma de escribirla (proceso comunicativo y cognitivo).

- Decide qué y cómo escribirlo (planeamiento motor).

- Corrige los errores que comete cuando esta escribiendo (anticipación).

- Reproduce las grafías de cada palabra que le dictan y comprueba que sean correctas o hace los cambios que sean necesarios cuando comete un error (CA).

- Muestra al profesor su trabajo (Cambio en el ambiente).

- Si su trabajo resulta correcto, continua en la actividad, de lo contrario hace las acomodaciones nece- sarias para corregirlo (retroalimentación).

Cuando el proceso es adecuado ninguna parte del cerebro trabaja sola para la producción del CA. Ayres planteaba que casi el $80 \%$ del cerebro está involucrado en la organización, procesamiento e interpretación de la información sensorial (Stock Kranowitz, 1998). Para lograr el CA, por ejemplo, el cerebro utiliza mecanismos como la Modulación, la Inhibición, la Habituación y la Facilitación de las funciones nerviosas que involucran muchas estructuras y procesos cerebrales:

La Modulación se refiere a la capacidad que tiene el cerebro de regular su nivel de actividad. Esta influye sobre el comportamiento físico, emocional y mental necesario para llevar a cabo una actividad. En el ejemplo del dictado, el mecanismo de Modulación, se observa en el nivel de atención y el manejo de los estímulos sensoriales que hace el niño, para mantenerse organizado e interesado durante el dictado.

La Inhibición se refiere a la capacidad del Sistema Nervioso de eliminar los impulsos innecesarios o irrelevantes que provienen de los estímulos ambientales los cuales pueden interferir con la ejecución de la tarea. El ejemplo del mecanismo de Inhibición, se observa en la capacidad de ignorar la sensación, proveniente de la forma del lápiz o la temperatura de la hoja de papel, que tiene el niño mientras toma el dictado, logrando asi su atención a los estímulos relevantes.

La Habituación se refiere a la capacidad del Cerebro de reconocer y ma- 
nejar impulsos provenientes de estimulos familiares, a los que no necesita atender tan especialmente porque pueden ser manejados de manera automática. El ejemplo del mecanismo de Habituación se da cuando el niño puede poner toda su atención en el dictado, porque no tiene que preocuparse por la posición del cuerpo en la silla o el tipo de agarre del lápiz, mientras escribe.

La Facilitación de las conexiones que trasladan impulsos sensoriales y motores, se refiere a la prioridad de los impulsos importantes o vitales para la ejecución de la tarea. El ejemplo del mecanismo de Facilitación se refiere a la atención que pone el niño a la voz del profesor y a lo que está escribiendo, aunque se escuchen otros niños dentro del aula.

\section{Cuando se presentan problemas:}

Entre más eficiente sea el balance entre los mecanismos cerebrales mejor será el CA. Pero si se presenta un funcionamiento deficiente de alguno de esos mecanismos se producirán dificultades. El cerebro no será eficiente para procesar la información causando retrasos en la relación e interpretación de la información para su uso. El resultado de estos problemas se parece a cuando se presenta un gran trancón de carros en una avenida, por ese motivo todas las personas llegarán tarde a trabajar y todas las actividades del día se retrasaran.

Cuando el proceso de IS no es eficiente se pueden presentar problemas de Atención. Muchos autores como Mulligan en 1998,Cermak en 1988, Holborow y Oetter en 1986, han estudiado la relación entre los problemas de IS y los de Atención. Por ejemplo Shelly Mulligan (13), en su estudio sobre los patrones de respuestas sensoriomotoras que presentan los niños con problemas de atención, se refirió a la relación entre los problemas de atención y los mecanismos neurológicos descritos anteriormente. Los problemas de atención pueden ser debidos a problemas en la Modulación, Inhibición, Facilitación o Habituación, ya que la atención también depende de la organización y eficiencia de esos mecanismos.

Mulligan basa este planteamiento en los estudios de Copeland en 1995, quien expresó que los problemas de atención se consideran desordenes neurofisiológicos debidos a una disfunción del Sistema Nervioso Central, tanto a nivel neuroanatómico como neuroquímico.

Por otra parte, Stock Kranowitz(15) describe otras razones que pueden influir en el adecuado funcionamiento del cerebro como son: la pobre acción de los impulsos sensoriales, la desorganización neurológica o la escasa respuesta ante las exigencias ambientales:

- Si el impulso sensorial es interrumpido en el cerebro, no se obtendrá la suficiente información sobre la situación o se presentará exceso de un solo tipo. En el ejemplo del dictado, si el niño tiene problemas para modular la información sensorial. Sentirá por ejemplo, fastidio con la textura del papel, por lo que no podrá séguir escribiendo.

- Cuando hay desorganización, el cerebro no recibe toda la informa- 
ción o esta se hace inconsistente y variable, lo cual no le permitirá seleccionarla y relacionarla para que sea interpretada. Por ejemplo, el niño tendrá problemas para oír lo que está dictando el profesor pues no logrará dejar de escuchar a un compañero que habla, así que no podrá copiar la palabra correcta.

- Cuando la respuesta es escasa a nivel motor, emocional o mental, el niño no tendrá un buen patrón para recordar las grafías de las letras y no podrá utilizar mecanismos como la anticipación o la retroalimentación para corregir lo que escribe.

Con relación al impacto de la desorganización neurológica sobre los periodos de atención, Mulligan también describe los estudios de Sharon Cermak en 1991. Ella encontró que muchos niños con dificultades de atención tienen problemas de IS y presen- tan por ejemplo, fallas de equilibrio, coordinación motora, percepción visomotriz y de planeamiento motor. Los problemas de IS afectan el desempeño general del niño lo cual incluye su habilidad para atender, aprender, organizarse y mantener un nivel de actividad acorde con la situación.

Estos planteamientos también fueron comprobados por Fisher y Murray (7), quienes proponen que los problemas de atención son el resultado de un deficiente procesamiento de la información sensorial: Táctil, Vestibular, Propioceptiva, Visual y Auditiva.

En conclusión, los problemas de IS llevan al niño a presentar un deficiente $\mathrm{CA}$ que trae consigo problemas de atención. Por estas razones el niño no podrá responder de manera eficiente ante las situaciones y por lo tanto su desempeño no será exitoso.

\section{Relación entre el ambiente, los procesos sensoriomotores, ejecutivos, $y$ las habilidades para atender y responder adaptativamente}

El desarrollo sensoriomotor y ejecutivo (DSE), se puede explicar como una suma de procesos que favorecen la ejecución de habilidades para el desempeño ocupacional. Estas habilidades se desarrollan principalmente durante los ocho primeros años. Cada adquisición es vital para el aprendizaje de otra más compleja que a su vez permitirá un desempeño más maduro y evolucionado.
En la literatura autores como Willianson en 1984, Fisher y Bundy en 1992 o Carol Stock Kranowitz en 1998, describen esta evolución como una espiral que parte de comportamientos elementales hasta llegar a la ejecución de conductas maduras de alto nivel. Por ejemplo Stock Kranowitz (15) compara al desarrollo de habilidades con una construcción de "bloques" que conforman la estructura del ser humano. 
Todos los pisos o niveles de desarrollo son determinados por funciones del sistema nervioso las cuales tienen sus centros de relación, asociación e interpretación en diferentes sitios. Los pisos del edificio son los siguientes:

- Un nivel inicial que se puede considerar como el "terreno y los cimientos del edificio", conformado por el ambiente: situaciones, personas y objetos, entorno social, cultura y costumbres.

- El primer piso en el cual se desarrollan los sistemas sensoriales básicos: táctil, propioceptivo, vestibular, visual y auditivo.

- El segundo piso en el cual se desarrollan las habilidades corporales: la percepción corporal, el desarrollo postural, la bilateralidad y la especialización motriz.

- El tercer piso, donde se desarrollan las habilidades perceptivo-motoras: el desarrollo motor, la percepción del espacio y el tiempo y la percepción auditiva.

- El cuarto piso donde se desarrollan las habilidades cognitivas: el pensamiento y el lenguaje.

- El último piso donde se desarrollan las habilidades académicas y ejecutivas: el autocontrol, la regulación de los periodos de atención, las habilidades ejecutivas y las habilidades académicas.

Muchos autores como Bisell en 1988, Fisher y Murray en 1991, Dunn y De Gangi en 1992, Bennet y Dunn en
1994, Mulligan en 1996 y Ermer y Dunn en 1998, han descrito la relación entre el desarrollo de los procesos anteriormente enumerados, la capacidad para atender y la de responder en forma adaptativa al ambiente. Por ejemplo, Ermer y Dunn (6), en 1998 hicieron un estudio de la influencia de estos procesos sobre el desarrollo normal partiendo del análisis del comportamiento de los niños con disfunciones sensoriales que presentaban desórdenes de desatención con Hiperactividad.

En su estudio encontraron que estos niños tienen dificultades en procesar y manejar estímulos sensoriales provenientes del ambiente, mostrando conductas de desatención e hiperactividad, malestar sensorial y reacciones emocionales adversas ante los estímulos. A continuación se describirán las dificultades que presentan teniendo en cuenta la organización de los niveles del desarrollo descritos con anterioridad.

El ambiente se refiere a las condiciones, situaciones y personas que rodean al individuo en cada contexto $y$ que determinan de manera importante sus opciones para desempeñarse de manera exitosa (Richter y Oetter, 1990). También es el espacio donde el niño utiliza las habilidades sensoriomotoras para manipular los elementos o moverse adecuadamente de acuerdo con la situación y los hábitos propios de cada actividad. En otras palabras, la forma de actuar en el rol que asume, como por ejemplo el de escolar, el de juego, o el social. Además crea estrategias para entender el mundo que le rodea y construir conocimiento. 
El ambiente ofrece relaciones cambiantes con las personas, situaciones y objetos, las cuales son mas complejas en la medida que el niño amplia su relación con el entorno. Además éste tiene un importante componente cultural que depende del contexto donde el niño crece.

Ritcher y Oetter (14), proponen que el ambiente permite al niño crear un "Alfabeto Básico" para lograr su adaptación al contexto sociocultural. Este alfabeto responde a los valores, creencias y costumbres. Además, parte de la interacción del niño con las experiencias sensoriales, motoras, comunicativas, afectivas y cognitivas, las cuales favorecen la apropiación de lo que le rodea y la interacción con las personas.

Cuando un niño tiene dificultades para adaptarse al ambiente que le rodea no puede actuar de manera competente ante la situación (Richter y Oetter, 1990). Es como si existiera un desequilibrio entre lo que el niño es capaz de hacer y lo que se espera de él. Por lo tanto puede presentar dificultades atención, falta de interés, angustia, irritabilidad, sobreestimulación, pobre responsividad ante los estímulos ambientales, o problemas cognitivos.

Por otra parte, la dificultad para adaptarse al ambiente conduce a un circulo vicioso que no permite el desarrollo de las habilidades sensoriomotoras y ejecutivas, que se describirán a continuación.

La información táctil se refiere a la percepción de sensaciones a través de la piel tales como tacto, temperatura, o texturas (Bissell, 1988). Su papel consiste en favorecer el desarrollo del esquema corporal que es la base de la habilidad motora pues el niño aprende a conocer cómo es y cómo utilizar su cuerpo. Esta capacidad es necesaria, por ejemplo, para manejar elementos escolares como el lápiz o las tijeras. Además la información táctil permite percibir las diversas texturas y aceptar su contacto, como por ejemplo las que ofrecen las prendas de vestir.

Los desordenes táctiles se han relacionado con los problemas de atención (Mulligan, 1996; Dunn y DeGangi, 1992). Cuando se presentan problemas en la percepción táctil, el niño dedica demasiada atención a este sistema y no es eficiente en su trabajo. Por ejemplo, no puede atender a lo que le están diciendo que haga. No escucha a su profesora porque está tratando de manejar las tijeras que están frías o se está quitando la ropa porque le pica.

La madurez propioceptiva se refiere a la información proveniente de músculos y articulaciones para percibir la pisicón y el movimiento de las partes del cuerpo. También participa el la conciencia del peso del cuerpo y de los objetos (Bisell, 1988). La madurez vestibular procesa la sensación que se tiene del movimiento en el espacio y tiene que ver con el control del equilibrio y el sentido de dirección (Bisell, 1988).

Estas sensaciones inflùyen por èjemplo, sobre el desarrollo de la postura necesaria para sentarse adecuada mente y utilizar de la mejor manera las manos para realizar las actividades durante la clase. 
Gracias a la información propioceptiva y vestibular es posible para el niño mantener el equilibrio en la silla sin necesidad de usar las manos para sostenerse. De esta manera las deja libres para usar el lápiz. Además este tipo de información, permite desarrollar el rango de fuerza necesario para sostener el lápiz y hacer que el trazo salga con la adecuada presión: ni tan suave que no se vea, ni tan duro que traspase varias hojas.

Los desordenes posturales se han relacionado con los problemas de atención (Mulligan, 1996; Dunn y DeGangi, 1992). Por ejemplo cuando el niño tiene problemas propioceptivos y vestibulares, no puede atender a lo que está sucediendo a su alrededor ya que está preocupado porque se puede caer, o porque no sabe cuánta fuerza hacer con el lápiz. Por estar controlando esas cosas no sabe lo que le están dictando.

\section{La percepción de la corporalidad pro-} viene de la sensación que da la piel, los músculos y las articulaciones, o percepción sensoriomotora. Esto le permite al cerebro crear una imagen corporal, conocer cómo funciona el cuerpo y tener una idea del yo individual a partir del esquema corporal Esto le permite al cerebro saber qué parte del cuerpo se mueve, cómo lo hace y qué posición asume (Bisell, 1988). Gracias a esto, el niño puede hacer los ajustes necesarios para que el movimiento cumpla con el objetivo que se propone. Por ejemplo, ir hasta la caneca del salón para botar un papel arrugado $\sin$ estrellarse con ningún compañero o pupitre.
Las fallas de conocimiento corporal se asocian con problemas de atención (Fisher y Murray, 1992). Un niño con pobre percepción corporal tendrá dificultades en saber cómo es su cuerpo o cómo moverlo para cumplir con un patrón motor. Seguramente se va a estrellar con un pupitre y va a regar los elementos escolares de un compañero.

\section{El desarrollo de la bilateralidad se re-} fiere a la habilidad de combinar los dos lados del cuerpo en los patrones motores, de cruzar su línea media y de realizar tareas de hacer y sostener (Bisell, 1988). Por ejemplo, gracias a esto, el niño puede usar una mano para sostener el papel y la otra para escribir sin mover el cuerpo.

Muchos niños con problemas de atención tienen dificultades para realizar acciones de coordinación bilateral (Mulligan, 1996). Cuando tienen esta dificultad no pueden manejar sus dos manos al tiempo o no cruzan la línea media y tienen que acomodar el cuerpo o el trabajo para ejecutarlo.

El procesamiento auditivo es la habilidad de percibir y entender lo que es escuchado. Esta habilidad incluye la capacidad de discriminar los sonidos, de entenderlos, recordarlos o de asociarlos (Bisell, 1988). Esto permite al niño, por ejemplo, entender las instrucciones que da el profesor en clase y ejecutarlas tan pronto las escucha.

Bisell (2) plantea que los problemas de procesamiento auditivo influyen sobre la habilidad para atender. El niño puede lucir confundido o desatento y ne- 
cesitar mas tiempo para ejecutar las instrucciones que se le dan, o requerir que estas le sean repetidas.

El procesamiento visual es descrito por Bisell (2) como el proceso de extraer y organizar la información proveniente del ambiente para entender lo que se ve. Algunas habilidades de la percepción visual están presentes al nacer, pero otras no se desarrollan completamente sino hasta la adolescencia.

Las adecuadas habilidades de percepción visual le permiten al niño organizar conceptos espaciales que le dan un sentido de ubicación de su cuerpo con respecto al espacio que le rodea. A esta organización se le denomina direccionalidad (Dunn y DeGangi, 1992). Además la percepción visual favorece la comprensión del concepto de derecha-izquierda, el cual está ligado a la pércepción de los dos lados del cuerpo. Gracias a esto el niño puede, por ejemplo, juzgar la distancia y dirección entre él y el compañero al que debe lanzar un balón.

Los problemas de percepción visual se han relacionado con los de atención ya que el niño no puede concentrarse en el estímulo visual importante y todo el ambiente visual es motivo de distracción (Mulligan, 1996; Dunn y DeGangi, 1992). Cuando se presentan problemas en de percepción visual, por ejemplo, el niño no puede lanzar el balón en la dirección correcta o no le imprime la fuerza necesaria para que llegue hasta el otro niño. O tal vez se queda mirando otra cosa y se olvida de lanzar el balón.
Durante los primeros siete años se desarrolla el proceso de especialización hemisférica para el comando de funciones del lenguaje, la percepción visual, las capacidades cognitivas, el control emocional y el control de las acciones motoras (Fisher y Murray, 1991). Fisher plantea que el $95 \%$ de la humanidad tiene una preferencia del Hemisferio Izquierdo para las funciones del lenguaje. El Hemisferio Izquierdo asume funciones como producir y procesar el lenguaje, reconocer las letras en una palabra, leer y comprender lo que se lee, recordar lo que se oye. Por su parte el Hemisferio Derecho tiene funciones como procesar la información visual, desarrollar la percepción visual y la memoria no verbal.

Cuando el niño tiene una buena lateralización de funciones puede, por ejemplo, manejar con gran facilidad la información visual y auditiva que recibe durante la clase.

Muchos problemas de atención se deben a una pobre Lateralización de las funciones Hemisféricas (Fisher y Murray, 1992). Cuando esto sucede, el niño no puede manejar de manera eficiente la información de lo que le dicen o lo que ve, ya que no logra procesarla de manera rápida.

La Especialización motriz se refiere al establecimiento de la preferencia: un lado del cuerpo se especializa para ejecutar las tareas motoras y el otro lado es el que ayuda y / o sostiene los objetos. Puede ser asumida por cualquiera de los Hemisferios Cerebrales estableciendo la lateralidad derecha o izquierda (Bisell, 1986). 
Cuando se presentan problemas de lateralidad se afecta la habilidad manual. Por ejemplo, un niño que no tiene definida la mano dominante, se demora escogiendo qué mano va a escribir o cambiando de mano mientras lo hace. De pronto no escucha lo que hay que hacer.

El Control motor fino se refiere a la habilidad manual para realizar patrones de manipulación para acciones precisas y coordinadas (Bisell, 1986). Para realizar actividades de motricidad fina es importante la coordinación de los ojos con los movimientos de las manos. Gracias al control motor fino es posible manejar elementos como el lápiz, las tijeras o los cubiertos.

La habilidad motora es la capacidad que tiene el individuo de concebir, planear y ejecutar acciones motoras específicas. Esta es el resultado de unos adecuados mecanismos de IS los cuales permiten escoger los patrones motores correctos para interactuar con el ambiente. Esto le da al niño habilidad y destreza para desempeñar sus actividades (Fisher y Bundy, 1994).

Con la práctica de los patrones motores básicos y los de manipulación, el niño estará en capacidad de controlar con habilidad elementos como el lápiz, las tijeras o el borrador. Este proceso toma alrededor de 7 años, ya que los finos movimientos de la mano, sólo son precisos a esta edad que es cuando se alcanza el control de todos sus pequeños músculos y articulaciones.

Por ejemplo, el niño tiene que ser capaz de sostener el lápiz con tres de- dos que se mueven suavemente para producir los trazos, mientras los otros dos están estáticos y flexionados dando apoyo a la mano sobre el papel. Además, una vez el niño ha desarrollado el planeamiento motor para repetir cada grafía, tiene que dedicar mucho tiempo a su práctica. Esto le dará la destreza necesaria para la copia o el dictado.

Las Habilidades de autocontrolse refieren a la capacidad de atender, organizarse y hacer el trabajo de manera autónoma. Esto favorece un desempeño ocupacional exitoso que parte de la propia escala de valores y el deseo de interactuar con el entorno (Kielhofner, 1992). En los primeros años de vida escolar se empiezan a moldear las capacidades para lograr la organización autónoma. Gracias a estas habilidades, por ejemplo, un niño de 8 años puede alistar los elementos de trabajo que necesita para escribir como son lápiz, borrador, papel. Puede también ser mas independiente para terminar un trabajo con mínima supervisión ya que esta mejorando su capacidad de atender.

Los componentes del autocontrol son: organización, independencia y atención. Se desarrollan de manera progresiva durante los nueve primeros años y son esenciales para que el niño esté en capacidad de aprender lo que se le enseña.

Frances Ilg (9), cita a Reynell quien describe el desarrollo de la habilidad de atender como una secuencia: Se inicia al año de edad con una atención lábil. Pasa por un período de aten- 
ción rígida escogida por el niño hacia los dos años. A los tres años el niño tiene una atención de un solo canal visual o auditivo, controlada por el adulto. A los cuatro años el niño ya puede controlar ese canal por sí mismo. A los cinco años el niño empieza a manejar dos canales de atención al tiempo. A los seis años tiene una atención consistente, manteniéndola por períodos apropiados.

Por este motivo es importante considerar la edad del niño cuando se escoge el trabajo que va a realizar en clase, su duración, el tipo de instrucciones, el momento en que se dan, y el grado de exigencia que se le puede hacer.

Cuando un niño tiene dificultades para atender, no puede manejar de manera eficiente los canales y tiempo de atención. Seguramente estará funcionando en un nivel inferior de la secuencia descrita anteriormente. Por lo tanto no podrá, por ejemplo, recordar y ejecutar las instrucciones dadas para el trabajo.

La habilidad para solucionar problemas tiene que ver con la capacidad de analizar la situación, diseñar una estrategia de solución a la misma y ejecutar el plan para resolverla (Bisell, 1988).

Por ejemplo aprender a leer y escribir demanda del niño la capacidad de alistar los útiles escolares; su permanencia en la actividad para hacer la decodificación de lo que lee; tener la habilidad de poder iniciar y terminar la tarea por iniciativa propia; y la posibilidad de solucionar algunos de los problemas que surjan con autonomía.
Cuando un niño tiene problemas de atención, su habilidad de resolver problemas no es buena ya que no siempre cuenta con toda la información para analizar la situación y actuar de manera adaptativa (Bisell, 1988). Por ejemplo, si el niño tiene que copiar la tarea del tablero y se distrae escuchando lo que dice otro compañero, seguramente no se va a dar cuenta de que no ha escrito todo lo que la profesora escribió. Es probable que en la casa, no pueda descifrar la tarea con las pocas palabras que haya escrito $y$ no recuerde qué era lo que había que hacer. Por lo tanto no hará la tarea y obtendrá una mala calificación.

El Desarrollo emocional permite al nin̄o tener relaciones significativas con las personas, a partir del autoconocimiento, ef reconocimiento del otro y el sentido de grupo. Gracias a estas habilidades, las personas pueden expresar y reconocer sus emociones y las de los demás; actuar de acuerdo con su escala de valores; resolver los conflictos y pensar en las necesidades propias y las de su comunidad (Amaya, Restrepo y otras, 1998).

En su propuesta sobre la enseñanza de las habilidades para el desempeño emocional, Amaya y colaboradoras(1), plantean que cuando el niño tiene problemas de atención no logra desarrollar su madurez emocional. Por ejemplo no tiene la capacidad de "leer las claves emocionales en los demás" o reconocer los propios estados de ánimo y por lo tanto no logra expresarse de acuerdo con lo esperado para la situación. Si está jugando fútbol no tiene en cuenta lo que le está diciendo otro niño, ya que no lo escucha por- 
que está pendiente sólo del balón, por eso puede herir sus sentimientos sin darse cuenta y meterse en una pelea.

Dunn (5) plantea que los niños desarrollan habilidades para aprender a partir de un estilo cognitivo basado en fortalezas perceptuales de tipo táctil, propioceptivo, visual y / o auditivo. Este estilo se va desarrollando a través de los años: antes de los siete años, un niño aprende más fácilmente por medio del tacto y del movimiento corporal y se le dificultan las tareas de tipo visual y auditivo. Hacia los nueve años los niños se vuelven más visuales y se les facilita aprender a partir de lo que leen o de las imágenes y esquemas. A los doce años aprenden con facilidad a partir de la información auditiva y pueden recordar con facilldad lo que deben saber solo con el apoyo de lo que escuchan.

Además, cada persona tiene un estilo para aprender que se basa no sólo en sus fortalezas perceptuales sino en sus capacidades cognitivas y el tipo de ambiente donde está aprendiendo (Dunn, 1992). Por ejemplo algunos individuos recuerdan con facilidad lo que han visto; otros lo que han escuchado y otros lo que han experimentado y manipulado. Este estilo cognitivo resulta del procesamiento simultáneo de los siguientes elementos: El ambiente físico; el estado de ánimo; las características de las personas con que se estudia; El desarrollo físico y las capacidades cognitivas: Nivel de pensamiento, habilidad para solucionar problemas, estrategias para analizar la información, especialización hemisférica.
Cuando un niño tiene dificultades en las habilidades descritas anteriormente no tiene un estilo cognitivo eficiente para aprender, no consigue adaptarse al ambiente donde está estudiando ni logra manejar la información que ve, escucha, ensaya o manipula (Dunn y DeGangi, 1992). Esto puede hacer que luzca desatento ya que no encuentra estrategias apropiadas para aprender.

Por este motivo es que los maestros encuentran en el aula, niños que no funcionan con las estrategias que ellos plantean para acceder al conocimiento. A veces la propuesta sólo ofrece un estilo cognitivo y no acciones diversas que se acomoden a las necesidades y fortalezas de cada niño.

Para resumir, existe una clara relación entre el desarrollo de los procesos sensoriomotores, los ejecutivos y la capacidad para atender y responder en forma adaptativa al ambiente. Cuando estos procesos no evolucionan dentro de los parámetros esperados el niño tiene dificultades en procesar y manejar estímulos sensoriales provenientes del ambiente, mostrando conductas de desatención e hiperactividad.

Cuando un niño tiene dificultades para adaptarse al ambiente que le rodea no puede actuar de manera competente ante la situación. Es como si existiera un desequilibrio entre lo que el niño es capaz de hacer y lo que se espera de él. Por otra parte, esta dificultad conduce a un circulo vicioso que no permite el desarrollo de las habilidades sensoriomotoras y de ejecución. 


\section{Algunas evaluaciones del desempeño del niño $y$ del entorno que se utilizan en terapia ocupacional}

En terapia ocupacional se han propuesto varias evaluaciones para establecer tanto la madurez y organización de los procesos sensoriomotores como las funciones ejecutivas. También se han hecho algunas propuestas de evaluación ambiental. A continuación se hace un breve resumen de las más frecuentemente utilizadas y de las más importantes:

La evaluación de la madurez sensoriomotora y ejecutiva a través de la aplicación de pruebas-estandarizadas como el Test de Integración sensorial del Sur de California, diseñado por Jean Ayres en 1972, para identificar la tipologia de la disfunción de Integración sensorial en niños que presentaban problemas de atención, de comportamiento o de desempeño motor. A partir del test propuesto por Ayres se han hecho revisiones y adaptaciones. Por ejemplo las de Fisher, Bundy y Murray en 1991, quienes dieron gran importancia al uso de Observaciones Clínicas del desarrollo postural y el planeamiento motor para caracterizar mejor el diagnóstico.

Actualmente se utiliza la versión adaptada del Test de Integración sensorial del Sur de California (SIPT), la cual es aplicada por Terapeutas Ocupacionales que tienen una certificación que las acredita para aplicar e interpretar la evaluación. El test cuenta con 17 pruebas que permiten identificar los problemas de integración sensorial en niños de 4 a 9 años.

\section{El uso de cuestionarios y / o listas} de chequeo que permiten recolectar la información y consignar la información aportada por los padres y maestros del niño. Por ejemplo, en 1994, Rita Dunn (4), diseñó el Perfil Sensorial que permite identificar el comportamiento de los niños con y sin disfunciones ante las experiencias sensoriales que ofrecen las situaciones ambientales. EI perfil mide el impacto del proceso sensorial durante el desempeño de comportamientos de la vida diaria, con el fin de establecer cómo se regulan las sensaciones (Zuckerman, 1990; Dunn, 1994; Wilbarger, 1995).

La observación sistemática del niño en su ambiente la cual es necesaria para identificar las situaciones, espacios, objetos y personas que intervienen en el entorno del niño.

Eillen Ritcher y Patricia Oetter(14), en su artículo sobre la importancia del manejo de ambiente durante el tratamiento de Terapia Ocupacional, dicen que la evaluación del ambiente permite determinar las habilidades necesarias para seleccionar y organizar la información proveniente del entorno (qué, para qué, dónde, cómo, cuándo); estudiar el ambiente donde se desempeña el niño; establecer las demandas ambientales en el logro de una tarea (roles, forma de actuar, tiempo necesario para la ejecución de la tarea); determinar las tareas propias de un rol, un contexto cultural o una situación dada y delimitar los territorios 
- espacios para cada persona y cada tarea (lugar donde se realiza).

Esta evaluación pude realizarse a través de la entrevista a padres y maestros, con la utilización de listas de chequeo y con la observación de los ambientes en que se desempeña el niño (casa, colegio, parque, etc.).
Es de anotar que no fue posible encontrar en la literatura revisada algún instrumento estandarizado para realizar la evaluación ambiental ya que en general los autores revisados proponen solamente observaciones y / o descripciones sistemáticas de lo que se ve.

\section{La propuesta para realizar la evaluación de los procesos sensoriomotores y de ejecución que influyen sobre el comportamiento adaptativo y la atención}

Esta evaluación se basa en la experiencia de la autora y del grupo de trabajo del Jardín Infantil Por un Mañana, ubicado en Bogotá, Colombia. La propuesta de evaluación consiste en utilizar tres estrategias: una lista de chequeo del desempeño sensoriomotor del niño; la observación sistemática de los ambientes en que este se mueve y un cuestionario ambiental que permite conocer las caracteristicas y posibilidades del ambiente físico $y$ de personas en el que vive el niño. $A$ continuación se describen y ejemplifican las tres estrategias.

\section{La evaluación de la madurez sensoriomotora y de ejecución}

La propuesta de evaluación consiste en utilizar la lista de chequeo del Desempeño Sensoriomotor y Ejecutivo del Jardín Infantil Por un Mañana. Esta lista fue diseñada por la autora, para que sea diligenciada por los padres, maestros y / o terapeutas que observan al niño durante la jornada escolar o en la casa.

El objetivo es identificar los comportamientos que caracterizan el desempeño sensoriomotor y ejecutivo del niño. Está dividida en 10 áreas que describen comportamientos relacionados con el tipo de procesamiento sensoriomotor o ejecutivo. Estas áreas son: Modulación sensorial; Procesamiento táctil, Vestibular, Propioceptivo, Visual, y Auditivo; Habilidad motora; Especialización hemisférica; Autocontrol y Desarrollo emocional.

La Lista de Chequeo del Desempeño Sensoriomotor y Ejecutivo califica cada comportamiento adaptativo con uno de los cuatro criterios que aquí aparecen: La conducta no aplica a la edad o situación observada (NA); la conducta no se observa nunca $(N)$; la conducta se observa algunas veces (AV); la conducta se observa casi siempre (CS); la conducta se observa siempre (S). 
A continuación se encuentra el cuadro No. 1 el cual presenta tres áreas de la lista de chequeo a manea de ejemplo:

\begin{tabular}{|l|l|}
\hline & \\
\hline Modulación sensorial: \\
Es irritable, llora y se queja por diversos motivos. \\
Se cansa con facilidad y/o se pone irritable a medida que pasa \\
el día. \\
No soporta los ruidos y se queja de oír muy duro. \\
Tiene temor exagerado a las alturas y al movimiento. \\
Su estado anímico es variable durante el día. \\
Reacciona de forma diferente ante las mismas situaciones: a \\
veces risa, a veces llanto o a veces indiferencia. \\
Tiene estallidos de llanto, rabia o pataletas sin razones apa- \\
rentes. \\
Necesita rituales para no desesperarse: ambientes sin cam- \\
bios, rutinas exactas. \\
Rechaza de manera sistemática ciertos estímulos: tacto y mo. \\
vimiento especialmente. \\
TotaL \\
\hline Procesamiento táctil: \\
Rechaza el contacto con personas, objetos, material de traba- \\
jo, o ropa. \\
No le gusta que le laven el pelo, la cara o los dientes. \\
Responde con rabia o fastidio al ser tocado repentinamente. \\
Trata de alejar a las personas para evitar que lo alcen, saluden \\
O toquen. \\
Es excesivamente carinoso: abraza todo el tiempo, se acerca \\
demasiado o busca caricias. \\
Tiende a rechazar el contacto de la mano con la hoja de papel \\
al escribir. \\
No acepta todas las texturas en la ropa o el la comida: rechaza \\
lo áspero, lo suave, lo blando o lo duro, etc. \\
No le gusta quitarse el saco o los zapatos. \\
No le gusta ponerse el saco o pantalón largo. \\
Se quita todo el tiempo los zapatos. \\
Tiende a mantener la boca abierta para que los labios no se \\
toquen: no porque no pueda respirar por la nariz. \\
No logra identificar los objetos a través del tacto. \\
Se mueve mucho o esta inquieto mientras realiza actividades \\
sedentarias: pintar, leer, manipular objetos pequeños. \\
Evita las actividades húmedas o pegajosas: plastilina, arcilla, \\
pintura. \\
Llora o luce incómodo cuando se le untan las manos de pegante \\
o pintura. \\
No le importa tener la cara y las manos sucias o estar mo- \\
coso.
\end{tabular}




\begin{tabular}{|l|l|l|l|}
\hline No acepta tener las manos o cara sucia por unos segundos. & & & \\
Se hace masajes con ciertos juguetes o texturas. & & \\
Se lleva los objetos a la boca permanentemente. & & \\
Toral & & \\
\hline Procesamiento propioceptivo: & \\
No puede imitar posiciones o movimientos. \\
Se le dificulta aprender nuevas secuencias motoras: manejar \\
juguetes, aprender juegos, hacer letras o figuras, articular cla- \\
ramente el lenguaje. \\
No sabe en qué posición tiene las piernas o los brazos si no \\
los mira. \\
Hace más fuerza al escribir, colorear o manipular objetos. \\
Hace muy poca fuerza al escribir, colorear o manipular objetos. \\
Le pesan mucho las cosas: un vaso lleno, el jabón nuevo, los \\
juguetes. \\
Se cansa antes de terminar una actividad: una plana, un juego \\
motor, caminando, comiendo. \\
Se cae con frecuencia. \\
Se cansa con facilidad al mantener posiciones estáticas: sen \\
tado, parado, bocaabajo. \\
Se coge de los muebles o se recuesta en ellos para no caerse. \\
Es brusco al moverse. \\
Es torpe en su desempeño motor: al jugar, al manipular los \\
objetos, al realizar tareas escolares como cortar, borrar o es- \\
cribir. \\
Es lento para moverse. \\
Total
\end{tabular}

Cuadro No. 1. Ejemplo de la lista de chequeo del desempeño sensoriomotor.

La lista de chequeo está planteada de manera que la presencia de un comportamiento adaptativo signifique un signo de alerta en el área evaluada. La presencia ocasional de los comportamientos adaptativos, puede indicar que existen problemas en el desempeño del área evaluada. La presencia constante de los comportamientos adaptativos, expresa un problema del área que debe ser atendido.
El valor de esta prueba radica en que se aprovecha el conocimiento que tiene los padres y maestros del comportamiento del niño. Ellos logran identificar con exactitud algunos comportamientos que no se establecen en las evaluaciones estandarizadas existentes en la actualidad. 


\section{La observación sistemática de los ambientes}

Puede realizarse a través de la descripción de lo que se ve cuando se observa al niño durante la ejecución de sus actividades en los contextos en que se desempeña. Estos sitios pueden ser la casa, el colegio o el parque, entre otros. Para realizar la observación ambiental se propone la observación sistemática del ambiente por parte de un terapeuta ocupacional.

Para realizar la observación se aconseja realizar con anterioridad unos cuadros que contengan los aspectos a ser observados, de manera que se puedan diligenciar con la información que se obtiene de la observación (Tobón, 1998). De esta manera se logra una visión completa y fácil, en la que no se pierda detalle alguno de lo que se ve. Lo observado debe consignarse de manera sistemática y cuidadosa en los cuadros de Descripción del Desempeño Sensoriomotor y Eje- cutivo durante la realización de la actividad; Descripción del Ambiente y Demandas Ambientales de la Actividad. Estos cuadros fueron diseñados por la autora para los Módulos de aprendizaje para estudiantes de Terapia Ocupacional sobre Ocupación y desarrollo neuroevolutivo (16).

El cuadro de Descripción del Desempeño Sensoriomotor y Ejecutivo, permite establecer los requisitos sensoriomotores y ejecutivos de la actividad y la forma cómo el niño los desarrolla. Este debe diligenciarse: explicando cómo realiza la actividad el niño; identificando los comportamientos adaptativos a nivel sensorial y ejecutivo que éste emplea; dando todos los detalles de la actuación del niño en relación a la situación y su interacción con las personas y objetos. En el cuadro No. 2 se ejemplifica la observación del desempeño sensoriomotor y ejecutivo.
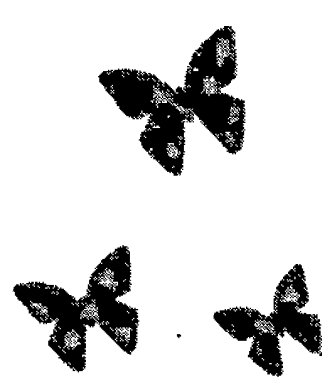


\begin{tabular}{|c|c|c|}
\hline $\begin{array}{l}\text { Nombre de la } \\
\text { actividad }\end{array}$ & Descripción & $\begin{array}{l}\text { Habilidades sensoriomotoras } \\
\text { y de desempeño observadas }\end{array}$ \\
\hline $\begin{array}{l}\text { Niño en clase } \\
\text { de Pintura. }\end{array}$ & $\begin{array}{l}\text { Esta pintando de pie frente } \\
\text { a un atril con un pincel. } \\
\text { Tiene las pinturas al lado } \\
\text { derecho sobre la mesa. } \\
\text { El profesor ordena los cam- } \\
\text { bios de color. } \\
\text { Algunos amigos están con- } \\
\text { versando. } \\
\text { El niño está concentrado en } \\
\text { su trabajo y muy contento } \\
\text { realizándolo. } \\
\text { Cuando se le unta la mano } \\
\text { de pintura, para de trabajar } \\
\text { y se la limpia. }\end{array}$ & $\begin{array}{l}\text { Estimulos Sensoriales: } \\
\text { Táctil en la mano al sentir el pincel. La } \\
\text { sensación de la ropa. } \\
\text { La pintura que se unta en la mano. } \\
\text { Vestibular y propioceptivo: La posición } \\
\text { en que esta. } \\
\text { La sensación de la posición de la mano. } \\
\text { Visual: Los colores que escoge. } \\
\text { La distancia del papel y el manejo ojo- } \\
\text { mano. } \\
\text { Auditivo: Las instrucciones del profesor } \\
\text { y lo que dicen los compañeros. } \\
\text { Desempeño Motor: } \\
\text { Percepción del cuerpo: La sensación de } \\
\text { la posición de cada parte del cuerpo. } \\
\text { Planeamiento motor: Los movimientos } \\
\text { que realiza con la mano en el papel y } \\
\text { para untar el pincel de pintura. } \\
\text { Coordinación de los dos lados del cuer- } \\
\text { po: Las dos manos trabajan. } \\
\text { Una sostiene el pincel y la otra el atril. } \\
\text { Especialización Hemisférica: Usa la } \\
\text { mano derecha para pintar y apoya la iz- } \\
\text { quierda en el caballete. } \\
\text { Autocontrol: Permanece en su trabajo a } \\
\text { pesar de los amigos que hablan. } \\
\text { No se detiene hasta terminar. } \\
\text { Desarrollo Emocional: Esta contento } \\
\text { pintando lo que la profesora le indica. } \\
\text { Estrategias cognitivas que usa: Esta } \\
\text { aprendiendo a partir de la experimenta- } \\
\text { ción con el movimiento. } \\
\text { Hay mucha información visual y auditiva. }\end{array}$ \\
\hline
\end{tabular}

Cuadro No. 2. Observación del Desempeño Sensoriomotor y Ejecutivo.

El cuadro de Descripción del Ambiente, puede establecer el ambiente humano y no humano que influye sobre el niño en el momento de realizar la tarea. También permite identificar las oportunidades que el entorno ofrece para hacer cambios y / o modificaciones que ayuden. Este debe diligenciarse explicando la situación que se observa; describiendo el espacio fisi- co donde se realiza la actividad; anotando las personas que interviene y su actitud durante la realización de la actividad; poniendo los objetos que se utilizan y dónde están ubicados; Indicando el tiempo que se emplea para la realización de la actividad. El cuadro No. 3 se refiere a la Descripción del Ambiente y cuenta con un ejemplo para ilustrarlo. 


\begin{tabular}{|c|c|c|c|c|c|}
\hline $\begin{array}{l}\text { Nombre } \\
\text { de la } \\
\text { actividad }\end{array}$ & $\begin{array}{l}\text { Descrip- } \\
\text { ción de la } \\
\text { situación }\end{array}$ & $\begin{array}{l}\text { Descripción } \\
\text { del espacio } \\
\text { físico o lugar } \\
\text { donde se } \\
\text { realiza la } \\
\text { actividad }\end{array}$ & $\begin{array}{l}\text { Personas que } \\
\text { intervienen y rol } \\
\text { que } \\
\text { asumen }\end{array}$ & $\begin{array}{l}\text { Objetos } \\
\text { que se } \\
\text { utilizan } \\
\text { durante la } \\
\text { actividad }\end{array}$ & $\begin{array}{c}\text { Tiempo } \\
\text { necesario } \\
\text { para } \\
\text { realizar la } \\
\text { actividad }\end{array}$ \\
\hline Almorzar & $\begin{array}{l}\text { El niño está } \\
\text { comiendo } \\
\text { una sopa en } \\
\text { el comedor. }\end{array}$ & $\begin{array}{l}\text { El niño está en } \\
\text { un comedor cua- } \\
\text { drado, con una } \\
\text { puerta, dos ven- } \\
\text { tanas a los la- } \\
\text { dos, que tiene } \\
\text { tres mesas. }\end{array}$ & $\begin{array}{l}\text { Una profesora que le } \\
\text { está enseñando a } \\
\text { agarrar bien la cu- } \\
\text { chara. } \\
\text { Dos compañeros que } \\
\text { están comiendo en la } \\
\text { misma mesa, quienes } \\
\text { están hablando de } \\
\text { una película. } \\
\text { Una niñera que viene } \\
\text { y va con la comida. }\end{array}$ & $\begin{array}{l}\text { Una cucha- } \\
\text { ra, el plato } \\
\text { de sopa. } \\
\text { Servilletas. } \\
\text { Mesa y silla. }\end{array}$ & $\begin{array}{l}\text { El niño utiliza } \\
\text { de } 5 \text { a } 10 \text { mi- } \\
\text { nutos para } \\
\text { tomarse } \\
\text { la sopa. }\end{array}$ \\
\hline
\end{tabular}

Cuadro No. 3. Descripción del ambiente.

El cuadro No. 3 se refiere a la Descripción de las Demandas Ambientales de la Actividad que realiza el niño. Con este cuadro se puede identificar lo que se espera del niño y los posibles problemas que se presentan. Además se pueden identificar soluciones que sean aplicables en el mismo ambiente donde se encuentra el niño. Para diligenciar el cuadro se tienen en cuenta qué se hace, para qué se realiza, dónde se realiza, cuándo sucede la actividad, y cómo se realiza esta. En el cuadro No. 4, que se encuentra a continuación, se desarrolla un ejemplo para su comprensión.

\begin{tabular}{|c|c|c|c|}
\hline $\begin{array}{c}\text { Nombre } \\
\text { de la tarea } \\
\text { OUE }\end{array}$ & $\begin{array}{c}\text { Conducta } \\
\text { adaptativa } \\
\text { esparada } \\
\text { PARA QUE }\end{array}$ & $\begin{array}{c}\text { Lugar donde se } \\
\text { realiza DONDE }\end{array}$ & $\begin{array}{c}\text { Hora y tiempo necesario } \\
\text { para su realización } \\
\text { CUANDO }\end{array}$ \\
\hline Almorzar & $\begin{array}{l}\text { El niño debe } \\
\text { aprender a } \\
\text { comer bien y } \\
\text { solo. }\end{array}$ & $\begin{array}{l}\text { En el comedor del colegio. } \\
\text { Sentado derecho frente a } \\
\text { su plato. Con compañeros } \\
\text { a los lados y al frente. }\end{array}$ & $\begin{array}{l}\text { A la hora del almuerzo. De 10a } \\
15 \text { minutos máximo. }\end{array}$ \\
\hline $\begin{array}{c}\text { Forma de } \\
\text { realizar la tarea } \\
\text { COMO } \\
\text { SENSORIAL }\end{array}$ & $\begin{array}{c}\text { Forma de } \\
\text { realizar la } \\
\text { tarea } \\
\text { como MOTOR }\end{array}$ & $\begin{array}{c}\text { Forma de realizar la } \\
\text { tarea } \\
\text { Como FUNCIONES } \\
\text { EJECUTIVAS }\end{array}$ & $\begin{array}{c}\text { Forma de realizar la tarea } \\
\text { COMO } \\
\text { CoGNITIVo Y } \\
\text { CoMUNICATIVO }\end{array}$ \\
\hline $\begin{array}{c}\text { Tactil: textura de } \\
\text { la comida, forma } \\
\text { y temperatura de } \\
\text { la cuchara. }\end{array}$ & $\begin{array}{l}\text { Posición del cuer- } \\
\text { po en la silla. } \\
\text { Patrón correcto } \\
\text { de agarre de la } \\
\text { cuchara. }\end{array}$ & $\begin{array}{l}\text { Atención a lo que dicen las } \\
\text { personas sin parar de } \\
\text { comer. }\end{array}$ & $\begin{array}{l}\text { Conocimiento del espacio del } \\
\text { comedor. } \\
\text { Conocimiento de los alimentos } \\
\text { que se comen. }\end{array}$ \\
\hline
\end{tabular}




\begin{tabular}{|c|c|c|c|}
\hline $\begin{array}{c}\text { Forma de realizar } \\
\text { la tarea } \\
\text { COMO } \\
\text { SENSORIAL }\end{array}$ & $\begin{array}{l}\text { Forma de } \\
\text { realizar la } \\
\text { tarea } \\
\text { COMO MOTOR }\end{array}$ & $\begin{array}{c}\text { Forma de realizar } \\
\text { la tarea } \\
\text { COMO FUNCIONES } \\
\text { EJECUTIVAS }\end{array}$ & $\begin{array}{c}\text { Forma de realizar la tarea } \\
\text { COMO } \\
\text { COGNITIVO Y } \\
\text { COMUNICATIVO }\end{array}$ \\
\hline $\begin{array}{l}\text { Sensación del } \\
\text { asiento y la superfi- } \\
\text { cie de la mesa. } \\
\text { Vestibular y } \\
\text { propioceptivo: } \\
\text { posición del cuerpo } \\
\text { en la silla. Altura de } \\
\text { la silla. Capacidad de } \\
\text { mantener la posición } \\
\text { sin caerse o } \\
\text { inctinarse sobre la } \\
\text { mesa. Visual: } \\
\text { Distancia de la sopa. } \\
\text { Colares de la comida } \\
\text { y la mesa. Movimien- } \\
\text { to de las personas. } \\
\text { Auditivo: Lo que } \\
\text { dicen las personas. } \\
\text { Ruidos ambientales. }\end{array}$ & $\begin{array}{l}\text { Patrón correcto } \\
\text { de uso de la cu- } \\
\text { chara (mano- } \\
\text { boca). } \\
\text { Correcta función } \\
\text { de alimentación } \\
\text { (masticación, } \\
\text { degusión). }\end{array}$ & $\begin{array}{l}\text { Acomodar la cuchara } \\
\text { para que no se riegue } \\
\text { la sopa. } \\
\text { Limpiarse con la servi- } \\
\text { lleta al chorrearse. } \\
\text { Saber cuando acabar. } \\
\text { Organización para } \\
\text { mantenerse en la acti- } \\
\text { vidad. }\end{array}$ & $\begin{array}{l}\text { Posibilidad de intervenir en la } \\
\text { conversación. } \\
\text { Entender las instrucciones que } \\
\text { se le dan. } \\
\text { Expresión verbal de lo que se } \\
\text { piensa. } \\
\text { Organización visoespacial } \\
\text { dentro del ambiente. }\end{array}$ \\
\hline
\end{tabular}

Cuadro No. 4. Demandas ambientales de la tarea.

\section{La elaboración de un cuestionario ambiental}

El Cuestionario Ambiental que se utiliza en el jardín Infantil Por un Mañana, fue diseñado por Tobón y Lizarazo en 1995. Su objetivo es conocer las caracteristicas y posibilidades del ambiente físico y de personas de la casa. Además busca establecer las opciones con que el ambiente cuenta e identifica la necesidad de hacer cambios. El cuestionario es diligenciado por los padres del niño, los cuales informan sobre:

- El ambiente físico: Descripción del cuarto del niño; de los espacios donde pone sus libros, juguetes, y ropa; sitios de la casa donde él pasa más tiempo; recursos de la co- munidad disponibles para el niño como bibliotecas, parques, sitios para la recreación, teatros, etc.

- El ambiente social: Descripción de las personas que interactúan con el niño cuando juega, estudia, realiza actividades recreativas y se desempeña en las tareas de autocuidado; amigos favoritos y personas con las que pasa la mayor parte del tiempo cuando está en la casa; actividades sociales recreativas y deportivas en las que el niño participa dentro de su comunidad o que estarian disponibles.

- Ambiente de toma de responsabilidades: Descripción de las tareas 
diarias que el niño realiza para cuidar de sí mismo y colaborar con los oficios del hogar como ordenar el cuarto, llevar la ropa sucia a un canasto, ordenar los juguetes, etc.

- Ambiente de estudio: Descripción de las rutinas y espacios donde el niño hace las tareas; las personas que le apoyan, motivan, valoran o supervisan su trabajo; los recursos que tiene a su alcance para hacer las tareas.

- Ambiente de juego: Descripción de los espacios, juegos y juguetes con los que cuenta el niño para jugar; horas en las que juega y tiempo que dura jugando; características de los juegos que prefiere $y / o$ evita; tipos de juego en los que participa cuando juega sólo, con amigos de su edad o con los adultos.

Luego de que los padres elaboran el cuestionario, se hace un análisis de la información para decidir el tipo de intervención en el contexto de la casa. Esta intervención se basa en consejos prácticos y fáciles de realizar durante la rutina cotidiana. Además se resalta aquello que los padres vienen realizando de manera adecuada y se enfatiza en la utilización de los recursos ambientales disponibles para beneficio del niño.

Para concluir, este articulo considera que la cuidadosa evaluación del desempeño sensoriomotor y ejecutivo y del ambiente permitirá identificar las fortalezas y debilidades tanto del niño como del entorno. Este conocimiento permitirá entender las razones que favorecen o limitan el desarrollo de una adecuada madurez sensoriomotora para enfrentar las exigencias ambientales y desempeñarse de manera adaptativa ante el ambiente.

También será posible establecer la necesidad de intervenir con estrategias que favorezcan la organización y el control del ambiente por parte del niño, con el fin de promover su ejecución ocupacional exitosa. Con esta visión integral, es posible que el niño con problemas de atención, encuentre el camino para lograr un desempeño adecuado en todas las actividades. 


\section{Bibliografía}

1. Amaya de Lee, Mariluces. B. Restrepo de Franco. C. De Prías. R. Lizarazo. M. Tobón (1999). Programa para el desarrollo emocional de los niños preescolares. Revista Ocupación Humana, 1999 8,1. Pag. 61-72

2. Bissell, Julie. J. Fisher. C. Owens. P. Polcyn. Sensory motor handbook. A guide for implementing and modifying activities in the classroom. Torrance, California (USA). Sensory Integration International. 1988

3. DiJoseph, Linda. L. Liotta-kleinfeld. Motor Behavior. En: Neuroscience and occupation. Links to practice. Charlotte Brassic, Editor. AOTA self study series. The American Occupational Therapy Association, Inc. Maryland, U.S.A. 1992. Cap. 7

4. Dunn, Winnie. C. Brown. Factor anaiysis on the Sensory Profile from a national sample of children without disabilities. The American Journa! of Occupational Therapy. 1997 July 7 August 51,7 Pag. 490-495

5. Dunn, Winnie. G. DeGangi. Sensory Integration and neurodevelopmental treatment for educational programming. En: Classroom aplications for school-based practice. Charlotte Brassic, Editor. AOTA self study series. Maryland (USA). The American Occupational Therapy Association, Inc. 1992. Cap. 3

6. Ermer, Julie. W. Dunn. The Sensory Profile: A discriminant analysis of children with and without disabilities. The American Journal of Occupational Therapy. 1998 April 52, 4 Pag. 283-289

7. Fisher Anne. E. Murray. Introduction to sensory integration theory. En:, Sensory Integration. Theory and practice. A. Fisher; A. Bundy. Murray, editoras. Philadelphia (USA). F. A. Davis Company. 1991

8. Gilfoyle, Elnora. A. Grady. J. Moore Children Adapt. A theory of sensoriomotor-sensary development. New York. (USA) Slack International Book Distribuitors. 1990

9. Ilg, Frances. L. Bates. Your two vears old child. (USA) Gessell Institute of Child Development. Delacote Press. 1976
10. Kielhofner, Gary The Model of Human Occupation. Conceptual foundations of Occupational Therapy. Philadelphia (USA) F. A. Davis Co 1994

11. McKeough, Michael The Nervous System as an information processing system. . En: Neuroscience and occupation. Links to practice. Charlotte Brassic, Editor. AOTA self study series. Maryland (USA). The American Occupational Therapy Association, Inc. 1992 Cap. 1

12. Mulligan, Shelley. An analysis of score patterns of children with attention disorders on the Sensory Integration and Praxis Tests. The American Journal of Occupational Therapy. 1996 September 50, 8. Pag. 647-653

13. Mulligan, Shelley, Patterns of sensory integration dysfunction: a confirmatory factor analysis. The American Journal of Occupational Therapy. 1998 Novenber / December 52, 10 Pag.

14. Richter, Eillen. P.Detter. Environmental matrices for sensory integrative traetment. En: Environment. Implications for Occupational therapy practice. Susan Cook, Editora. Maryland (USA).

The American Occupational Therapy Association, Inc. 1990

15. Stock Kranowitz, Carol. The out-of-sync child. New York (USA). Skylight Book. The Berkley Publishing Group. 1998

16. Tobón, Martha (1998). Dcupación y desarrollo neuroevolutivo. Módulos de aprendizaje para estudiantes de Terapia Dcupacional. Bogotá (Colombia) Ediciones rosaristas. 1998

17. (1998).

El juego, espacio natural para favorecer el desarrollo motor. Revista Ocupación Humana 1998 8,1.

Pag, 43-67

18. (2001).

Modulación sensorial un problema de sintonía. Revista Ocupación Humana, 2001 9, 1.

Pag. 57-71 